\title{
New concepts of fuzzy planar graphs
}

\author{
Sovan Samanta \\ Department of Applied \\ Mathematics with Oceanology \\ and Computer Programming, \\ Vidyasagar University, \\ Midnapore - 721 102, India. \\ email: ssamantavu@gmail.com
}

\author{
Anita Pal \\ Department of Mathematics, \\ National Institute of \\ Technology Durgapur, \\ Durgapur-713209, India. \\ e-mail: anita.buie@gmail.com
}

Madhumangal Pal

\author{
Department of Applied \\ Mathematics with Oceanology \\ and Computer Programming, \\ Vidyasagar University, \\ Midnapore - 721 102, India. \\ email: mmpalvu@gmail.com
}

\begin{abstract}
Fuzzy planar graph is an important subclass of fuzzy graph. Fuzzy planar graphs and its several properties are presented. A very close association of fuzzy planar graph is fuzzy dual graph. This is also defined and several properties of it are studied. Isomorphism on fuzzy graphs are well defined in literature. Isomorphic relation between fuzzy planar graph and its dual graph are established.
\end{abstract}

Keywords: Fuzzy graphs, fuzzy planar graphs, fuzzy dual graphs, isomorphism.

\section{INTRODUCTION}

Graph theory has vast applications in data mining, image segmentation, clustering, image capturing, networking, communication, planning, scheduling. For example, a data structure can be designed in the form of a tree which utilizes vertices and edges. Similarly, modeling of network topologies can be done using the concept of graph. In the same way, the most important concept of graph colouring is utilized in resource allocation, scheduling, etc. Also, paths, walks and circuits are used to solve many problems, viz. travelling salesman, database design, resource networking. This leads to the development of new algorithms and new theories that can be used in various applications.

There are many practical applications with a graph structure in which crossing between edges is a nuisance such as design problems for circuits, subways, utility lines, etc. Crossing of two connections normally means that the communication lines must be run at different heights. This is not a big issue for electrical wires, but it creates extra expenses for some types of lines, e.g. burying one subway tunnel under another. Circuits, in particular, are easier to manufacture if their connections can be constructed in fewer layers. These applications are designed by the concept of planar graphs. Circuits where crossing of lines is necessary, can not be represented by planar graphs. Numerous computational challenges including image segmentation or shape matching can be solved by means of cuts of planar graph.

After development of fuzzy graph theory by Rosenfeld [23], the fuzzy graph theory is increased with a large number of branches. McAllister [17] characterised the fuzzy intersection graphs. In this paper, fuzzy intersection graphs have been defined from the concept of intersection of fuzzy sets.
Samanta and Pal [25] introduced fuzzy tolerance graphs as the generalisation of fuzzy intersection graphs. They also defined fuzzy threshold graphs [26]. Fuzzy competition graphs [24] are another kind of fuzzy graphs which are the intersection of the fuzzy neighbourhoods of vertices of a fuzzy graph. Many works have been done on fuzzy sets as well as on fuzzy graphs [2], [3], [4], [5], [7], [8], [10], [11], [12], [13], [14], [15], [19]. Abdul-jabbar et al. [1] introduced the concept of fuzzy planar graph. In this paper, the crisp planar graph is considered and the membership values are assigned on vertices and edges. They also defined fuzzy dual graph as a straight forward way as crisp dual graph. Again, Nirmala and Dhanabal [22] defined special fuzzy planar graphs. The work presented in this paper is similar to the work presented in [1]. In these papers, the crossing of edges in fuzzy planar graph is not allowed. But, in our work, we define fuzzy planar graph in such a way that the crossing of edges is allowed. Also, we define the fuzzy planarity value which measures the amount of planarity of a fuzzy planar graph. These two concepts are new and no work has been done with these ideas. It is also shown that an image can be represented by a fuzzy planar graph and contraction of such image can be made with the help of fuzzy planar graph. The fuzzy multigraphs, fuzzy planar graphs and fuzzy dual graphs are illustrated by examples. Also, lot of results are presented for these graphs. These results have certain applications in subway tunnels, routes, oil/gas pipelines representation, etc.

\section{PREliminaries}

A finite graph is a graph $G=(V, E)$ such that $V$ and $E$ are finite sets. An infinite graph is one with an infinite set of vertices or edges or both. Most commonly in graph theory, it is implied that the graphs discussed are finite. A multigraph [6] is a graph that may contain multiple edges between any two vertices, but it does not contain any self loops. A graph can be drawn in many different ways. A graph may or may not be drawn on a plane without crossing of edges.

A drawing of a geometric representation of a graph on any surface such that no edges intersect is called embedding [6]. A graph $G$ is planar if it can be drawn in the plane with its edges only intersecting at vertices of $G$. So the graph is non-planar 
if it can not be drawn without crossing. A planar graph with cycles divides the plane into a set of regions, also called faces. The length of a face in a plane graph $G$ is the total length of the closed walk(s) in $G$ bounding the face. The portion of the plane lying outside a graph embedded in a plane is infinite region.

In graph theory, the dual graph of a given planar graph $G$ is a graph which has a vertex corresponding to each plane region of $G$, and the graph has an edge joining two neighboring regions for each edge in $G$, for a certain embedding of $G$.

A fuzzy set $A$ on an universal set $X$ is characterized by a mapping $m: X \rightarrow[0,1]$, which is called the membership function. A fuzzy set is denoted by $A=(X, m)$.

A fuzzy graph [23] $\xi=(V, \sigma, \mu)$ is a non-empty set $V$ together with a pair of functions $\sigma: V \rightarrow[0,1]$ and $\mu: V \times V \rightarrow$ $[0,1]$ such that for all $x, y \in V, \mu(x, y) \leq \min \{\sigma(x), \sigma(y)\}$, where $\sigma(x)$ and $\mu(x, y)$ represent the membership values of the vertex $x$ and of the edge $(x, y)$ in $\xi$ respectively. A loop at a vertex $x$ in a fuzzy graph is represented by $\mu(x, x) \neq 0$. An edge is non-trivial if $\mu(x, y) \neq 0$.

A fuzzy graph $\xi=(V, \sigma, \mu)$ is complete if $\mu(u, v)=$ $\min \{\sigma(u), \sigma(v)\}$ for all $u, v \in V$, where $(u, v)$ denotes the edge between the vertices $u$ and $v$.

Several definitions of strong edge are available in literature. Among them the definition of [9] is more suitable for our purpose. The definition is given below. For the fuzzy graph $\xi=(V, \sigma, \mu)$, an edge $(x, y)$ is called strong [9] if $\frac{1}{2} \min \{\sigma(x), \sigma(y)\} \leq \mu(x, y)$ and weak otherwise. The strength of the fuzzy edge $(x, y)$ is represented by the value $\mu(x, y)$ $\overline{\min \{\sigma(x), \sigma(y)\}}$

If an edge $(x, y)$ of a fuzzy graph satisfies the condition $\mu(x, y)=\min \{\sigma(x), \sigma(y)\}$, then this edge is called effective edge [21]. Two vertices are said to be effective adjacent if they are the end vertices of the same effective edge. Then the effective incident degree of a fuzzy graph is defined as number of effective incident edges on a vertex $v$. If all the edges of a fuzzy graph are effective, then the fuzzy graph becomes complete fuzzy graph. A pendent vertex in a fuzzy graph is defined as a vertex of an effective incident degree one. A fuzzy edge is called a fuzzy pendant edge [24], if one end vertex is fuzzy pendant vertex. The membership value of the pendant edge is the minimum among the membership values of the end vertices.

A homomorphism [20] between fuzzy graphs $\xi$ and $\xi^{\prime}$ is a map $h: S \rightarrow S^{\prime}$ which satisfies $\sigma(x) \leq \sigma^{\prime}(h(x))$ for all $x \in S$ and $\mu(x, y) \leq \mu^{\prime}(h(x), h(y))$ for all $x, y \in S$ where $S$ is set of vertices of $\xi$ and $S^{\prime}$ is that of $\xi^{\prime}$.

A weak isomorphism [20] between fuzzy graphs is a bijective homomorphism $h: S \rightarrow S^{\prime}$ which satisfies $\sigma(x)=$ $\sigma^{\prime}(h(x))$ for all $x \in S$.

A co-weak isomorphism [20] between fuzzy graphs is a bijective homomorphism $h: S \rightarrow S^{\prime}$ which satisfies $\mu(x, y)=$ $\mu^{\prime}(h(x), h(y))$ for all $x, y \in S$.

An isomorphism [20] between fuzzy graphs is a bijective homomorphism $h: S \rightarrow S^{\prime}$ which satisfies $\sigma(x)=\sigma^{\prime}(h(x))$ for all $x \in S$ and $\mu(x, y)=\mu^{\prime}(h(x), h(y))$ for all $x, y \in S$.
The underlying crisp graph of the fuzzy graph $\xi=(V, \sigma, \mu)$ is denoted as $\xi^{*}=\left(V, \sigma^{*}, \mu^{*}\right)$ where $\sigma^{*}=\{u \in V \mid \sigma(u)>0\}$ and $\mu^{*}=\{(u, v) \in V \times V \mid \mu(u, v)>0\}$.

A (crisp) multiset over a non-empty set $V$ is simply a mapping $d: V \rightarrow N$, where $N$ is the set of natural numbers. Yager [31] first discussed fuzzy multisets, although he used the term "fuzzy bag". An element of nonempty set $V$ may occur more than once with possibly the same or different membership values. A natural generalization of this interpretation of multiset leads to the notion of fuzzy multiset, or fuzzy bag, over a non-empty set $V$ as a mapping $\tilde{C}: V \times[0,1] \rightarrow N$. The membership values of $v \in V$ are denoted as $v_{\mu^{j}}, j=1,2, \ldots, p$ where $p=\max \left\{j: v_{\mu^{j}} \neq 0\right\}$. So the fuzzy multiset can be denoted as $M=\left\{\left(v, v_{\mu^{j}}\right), j=\right.$ $1,2, \ldots, p \mid v \in V\}$.

To define fuzzy planar graph, fuzzy multigraph is essential as planar graphs contain multi-edges. In the next section, fuzzy multigraph is defined.

\section{FUZZY MULTIGRAPH}

In this section, the fuzzy multigraph is defined.

Definition 1: Let $V$ be a non-empty set and $\sigma: V \rightarrow$ $[0,1]$ be a mapping. Also let $E=\left\{\left((x, y),(x, y)_{\mu^{j}}\right), j=\right.$ $\left.1,2, \ldots, p_{x y} \mid(x, y) \in V \times V\right\}$ be a fuzzy multiset of $V \times V$ such that $(x, y)_{\mu^{j}} \leq \min \{\sigma(x), \sigma(y)\}$ for all $j=$ $1,2, \ldots, p_{x y}$, where $p_{x y}=\max \left\{j \mid(x, y)_{\mu^{j}} \neq 0\right\}$. Then $\psi=(V, \sigma, E)$ is denoted as fuzzy multigraph where $\sigma(x)$ and $(x, y)_{\mu^{j}}$ represent the membership value of the vertex $x$ and the membership value of the edge $(x, y)$ in $\psi$ respectively.

It may be noted that there may be more than one edge between the vertices $x$ and $y$. $(x, y)_{\mu^{j}}$ denotes the membership value of the $j$-th edge between the vertices $x$ and $y$. Note that $p_{x y}$ represents the number of edges between the vertices $x$ and $y$.

\section{FUZZY PLANAR GRAPHS}

Planarity is important in connecting the wire lines, gas lines, water lines, printed circuit design, etc. But, some times little crossing may be accepted to these design of such lines/ circuits. So fuzzy planar graph is an important topic for these connections.

A crisp graph is called non-planar graph if there is at least one crossing between the edges for all possible geometrical representations of the graph. Let a crisp graph $G$ has a crossing for a certain geometrical representation between two edges $(a, b)$ and $(c, d)$. In fuzzy concept, we say that this two edges have membership values 1 . If we remove the edge $(c, d)$, the graph becomes planar. In fuzzy sense, we say that the edges $(a, b)$ and $(c, d)$ have membership values 1 and 0 respectively.

Let $\xi=(V, \sigma, \mu)$ be a fuzzy graph and for a certain geometric representation, the graph has only one crossing between two fuzzy edges $((w, x), \mu(w, x))$ and $((y, z), \mu(y, z))$. If $\mu(w, x)=1$ and $\mu(y, z)=0$, then we say that the fuzzy graph has no crossing. Similarly, if $\mu(w, x)$ has value near to 1 and $\mu(w, x)$ has value near to 0 , the crossing will not be important for the planarity. If $\mu(w, x)$ has value near to 1 and 
$\mathrm{a}(0.7)$

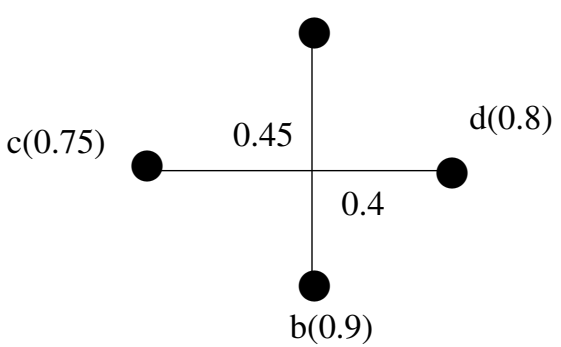

Fig. 1. Intersecting value between two intersecting edges.

$\mu(w, x)$ has value near to 1 , then the crossing becomes very important for the planarity.

Before going to the main definition, some co-related terms are discussed below.

\section{A. Intersecting value in fuzzy multigraph}

In fuzzy multigraph, when two edges intersect at a point, a value is assigned to that point in the following way. Let in a fuzzy multigraph $\psi=(V, \sigma, E), E$ contains two edges $\left((a, b),(a, b)_{\mu^{k}}\right)$ and $\left((c, d),(c, d)_{\mu^{l}}\right)$ which are intersected at a point $P$, where $k$ and $l$ are fixed integers.

Strength of the fuzzy edge $(a, b)$ can be measured by the value $I_{(a, b)}=\frac{(a, b)_{\mu^{k}}}{\min \{\sigma(a), \sigma(b)\}}$. If $I_{(a, b)} \geq 0.5$, then the fuzzy edge is called strong otherwise weak.

We define the intersecting value at the point $P$ by $\mathcal{I}_{P}=$ $\frac{I_{(a, b)}+I_{(c, d)}}{2}$. If the number of point of intersections in a fuzzy multigraph increases, planarity decreases. So for fuzzy multigraph, $\mathcal{I}_{P}$ is inversely proportional to the planarity. Based on this concept, a new terminology is introduced below for a fuzzy planar graph.

Definition 2: Let $\psi$ be a fuzzy multigraph and for a certain geometrical representation $P_{1}, P_{2}, \ldots, P_{z}$ be the points of intersections between the edges. $\psi$ is said to be fuzzy planar graph with fuzzy planarity value $f$, where

$$
f=\frac{1}{1+\left\{\mathcal{I}_{P_{1}}+\mathcal{I}_{P_{2}}+\ldots+\mathcal{I}_{P_{z}}\right\}} .
$$

It is obvious that $f$ is bounded and the range of $f$ is $0<f \leq 1$.

If there is no point of intersection for a certain geometrical representation of a fuzzy planar graph, then its fuzzy planarity value is 1 . In this case, the underlying crisp graph of this fuzzy graph is the crisp planar graph. If $f$ decreases, then the number of points of intersection between the edges increases and obviously the nature of planarity decreases. From this analogy, one can say that every fuzzy graph is a fuzzy planar graph with certain fuzzy planarity value.

Example 1: Here an example is given to calculate the intersecting value at the intersecting point between two edges. Two edges $(a, b)$ and $(c, d)$ are intersected where $\sigma(a)=$ $0.7, \sigma(b)=0.9, \sigma(c)=0.75, \sigma(0.8), \mu(a, b)=0.4, \mu(c, d)=$ 0.45 (see Fig. 1). Strength of the edge $(a, b)$ is $\frac{0.4}{0.7}=0.57$ and that of $(c, d)$ is $\frac{0.45}{0.75}=0.6$. Thus the intersecting value at the point is $\frac{0.57+0.6}{2}=0.585$.
Fuzzy planarity value for a fuzzy multigraph is calculated from the following theorem.

Theorem 1: Let $\psi$ be a fuzzy multigraph such that edge membership value of each intersecting edge is equal to the minimum of membership values of its end vertices. The fuzzy planarity value $f$ of $\psi$ is given by $f=\frac{1}{1+N_{p}}$, where $N_{p}$ is the number of point of intersections between the edges in $\psi$. Proof. Let $\psi=(V, \sigma, E)$ be a fuzzy multigraph such that edge membership values of each intersecting edge is equal to minimum of its vertex membership values. For the fuzzy multigraph, $(x, y)_{\mu^{j}}=\min \{\sigma(x), \sigma(y)\}$ for each intersecting edge $(x, y)$ and $j=1,2, \ldots, p_{x y}$.

Let $P_{1}, P_{2}, \ldots, P_{k}$, be the point of intersections between the edges in $\psi, k$ being an integer. For any intersecting edge $(a, b)$ in $\psi, I_{(a, b)}=\frac{(a, b)_{\mu}{ }^{j}}{\min \{\sigma(a), \sigma(b)\}}=1$. Therefore, for $P_{1}$, the point of intersection between the edges $(a, b)$ and $(c, d), \mathcal{I}_{P_{1}}$ is equals to $\frac{1+1}{2}=1$. Hence $\mathcal{I}_{P_{i}}=1$ for $i=1,2, \ldots, k$.

Now, $f=\frac{1}{1+\mathcal{I}_{P_{1}}+\mathcal{I}_{P_{2}}+\ldots+\mathcal{I}_{P_{k}}}=\frac{1}{1+(1+1+\ldots+1)}=\frac{1}{1+N_{p}}$, where $N_{p}$ is the number of point of intersections between the edges in $\psi$.

Definition 3: A fuzzy planar graph $\psi$ is called strong fuzzy planar graph if the fuzzy planarity value of the graph is greater than 0.5 .

The fuzzy planar graph of Example 5 is not strong fuzzy planar graph as its fuzzy planarity value is less than 0.5 .

Thus, depending on the fuzzy planarity value, the fuzzy planar graphs are divided into two groups namely, strong and weak fuzzy planar graphs.

Theorem 2: Let $\psi$ be a strong fuzzy planar graph. The number of point of intersections between strong edges in $\psi$ is at most one.

Proof. Let $\psi=(V, \sigma, E)$ be a strong fuzzy planar graph. Let, if possible, $\psi$ has at least two point of intersections $P_{1}$ and $P_{2}$ between two strong edges in $\psi$.

For any strong edge $\left((a, b),(a, b)_{\mu^{j}}\right), \quad(a, b)_{\mu^{j}} \geq$ $\frac{1}{2} \min \{\sigma(a), \sigma(b)\}$. So $I_{(a, b)} \geq 0.5$.

Thus for two intersecting strong edges $\left((a, b),(a, b)_{\mu^{j}}\right)$ and $\left((c, d),(c, d)_{\mu^{i}}\right), \frac{I_{(a, b)}+I_{(c, d)}}{2} \geq 0.5$, that is, $\mathcal{I}_{P_{1}} \geq 0.5$. Similarly, $\mathcal{I}_{P_{2}} \geq 0.5$. Then $1+\mathcal{I}_{P_{1}}+\mathcal{I}_{P_{2}} \geq 2$. Therefore, $f=\frac{1}{1+\mathcal{I}_{P_{1}}+\mathcal{I}_{P_{2}}} \leq 0.5$. It contradicts the fact that the fuzzy graph is a strong fuzzy planar graph.

So number of point of intersections between strong edges can not be two. It is clear that if the number of point of intersections of strong fuzzy edges increases, the fuzzy planarity value decreases. Similarly, if the number of point of intersection of strong edges is one, then the fuzzy planarity value $f>0.5$. Any fuzzy planar graph without any crossing between edges is a strong fuzzy planar graph. Thus, we conclude that the maximum number of point of intersections between the strong edges in $\psi$ is one.

Face of a planar graph is an important feature. We now introduce the fuzzy face of a fuzzy planar graph.

Fuzzy face in a fuzzy graph is a region bounded by fuzzy edges. Every fuzzy face is characterized by fuzzy edges in its boundary. If all the edges in the boundary of a fuzzy face 
have membership value 1 , it becomes crisp face. If one of such edges is removed or has membership value 0 , the fuzzy face does not exist. So the existence of a fuzzy face depends on the minimum value of strength of fuzzy edges in its boundary. A fuzzy face and its membership value are defined below.

Definition 4: Let $\psi=(V, \sigma, E)$ be a fuzzy planar graph and

$E=\left\{\left((x, y),(x, y)_{\mu^{j}}\right), j=1,2, \ldots, p_{x y} \mid(x, y) \in V \times\right.$ $V\}$ and $p_{x y}=\max \left\{j \mid(x, y)_{\mu^{j}} \neq 0\right\}$. A fuzzy face of $\psi$ is a region, bounded by the set of fuzzy edges $E^{\prime} \subset E$, of a geometric representation of $\psi$. The membership value of the fuzzy face is

$$
\min \left\{\frac{(x, y)_{\mu^{j}}}{\min \{\sigma(x), \sigma(y)\}}, j=1,2, \ldots, p_{x y} \mid(x, y) \in E^{\prime}\right\} .
$$

A fuzzy face is called strong fuzzy face if its membership value is greater than 0.5 , and weak face otherwise. Every fuzzy planar graph has an infinite region which is called outer fuzzy face. Other faces are called inner fuzzy faces.

Example 2: In Fig. $2, \quad F_{1}, F_{2}$ and $F_{3}$ are three fuzzy faces. $F_{1}$ is bounded by the edges $\left(\left(v_{1}, v_{2}\right), 0.5\right),\left(\left(v_{2}, v_{3}\right), 0.6\right),\left(\left(v_{1}, v_{3}\right), 0.55\right) \quad$ with membership value 0.833 . Similarly, $F_{2}$ is a fuzzy bounded face. $F_{3}$ is the outer fuzzy face with membership value 0.33 . So $F_{1}$ is a strong fuzzy face and $F_{2}, F_{3}$ are weak fuzzy faces.

Every strong fuzzy face has membership value greater than 0.5 . So every edge of a strong fuzzy face is a strong fuzzy edge.

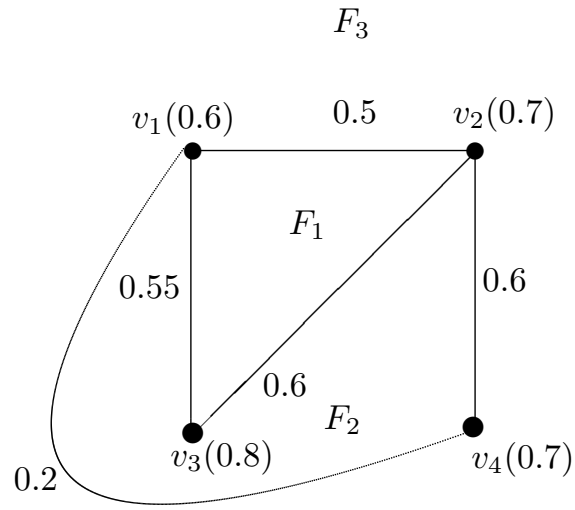

Fig. 2. Example of faces in fuzzy planar graph.

Theorem 3: If the fuzzy planarity value of a fuzzy planar graph is greater than 0.67 , then the graph does not contain any point of intersection between two strong edges.

Proof. Let $\psi=(V, \sigma, E)$ be a fuzzy planar graph with fuzzy planarity value $f$, where $f \geq 0.67$. Let, if possible, $P$ be a point of intersection between two strong fuzzy edges $\left((a, b),(a, b)_{\mu^{j}}\right)$ and $\left((c, d),(c, d)_{\mu^{j}}\right)$.

For any strong edge $\left((a, b),(a, b)_{\mu^{j}}\right), \quad(a, b)_{\mu^{j}} \geq$ $\frac{1}{2} \min \{\sigma(a), \sigma(b)\}$. Therefore, $I_{(a, b)} \geq 0.5$. For the minimum value of $I_{(a, b)}$ and $I_{(c, d)}, \mathcal{I}_{P}=0.5$ and $f=\frac{1}{1+0.5}<0.67$. A contradiction arises. Hence, $\psi$ does not contain any point of intersection between strong edges.

Motivated from this theorem, we introduce a special type of fuzzy planar graph called 0.67-fuzzy planar graph whose fuzzy planarity value is more than or equal to 0.67 . As in mentioned earlier, if the fuzzy planarity value is 1 , then the geometrical representation of fuzzy planar graph is similar to the crisp planar graph. It is shown in Theorem 7, if fuzzy planarity value is 0.67 , then there is no crossing between strong edges. For this case, if there is any point of intersection between edges, that is the crossing between the weak edge and any other edge. Again, the significance of weak edge is less compared to strong edges. Thus, 0.67-fuzzy planar graph is more significant. If fuzzy planarity value increases, then the geometrical structure of planar graph tends to crisp planar graph.

Any fuzzy planar graph without any point of intersection of fuzzy edges is a fuzzy planar graph with fuzzy planarity value 1 . Therefore, it is a 0.67 -fuzzy planar graph.

\section{FUZZY DUAL GRAPH}

We now introduce dual of 0.67-fuzzy planar graph. In fuzzy dual graph, vertices are corresponding to the strong fuzzy faces of the 0.67 -fuzzy planar graph and each fuzzy edge between two vertices is corresponding to each edge in the boundary between two faces of 0.67 -fuzzy planar graph. The formal definition is given below.

Definition 5: Let $\psi=(V, \sigma, E)$ be a 0.67 -fuzzy planar graph and $E=\left\{\left((x, y),(x, y)_{\mu^{j}}\right), j=1,2, \ldots, p_{x y} \mid(x, y) \in\right.$ $V \times V\}$. Again, let $F_{1}, F_{2}, \ldots, F_{k}$ be the strong fuzzy faces of $\psi$. The fuzzy dual graph of $\psi$ is a fuzzy planar graph $\psi^{\prime}=$ $\left(V^{\prime}, \sigma^{\prime}, E^{\prime}\right)$, where $V^{\prime}=\left\{x_{i}, i=1,2, \ldots, k\right\}$, and the vertex $x_{i}$ of $\psi^{\prime}$ is considered for the face $F_{i}$ of $\psi$.

The membership values of vertices are given by the mapping $\sigma^{\prime}: V^{\prime} \rightarrow[0,1]$ such that $\sigma^{\prime}\left(x_{i}\right)=\max \left\{(u, v)_{\mu^{j}}, j=\right.$ $1,2, \ldots, p_{u v} \mid(u, v)$ is an edge of the boundary of the strong fuzzy face $F_{i}$ \}.

Between two faces $F_{i}$ and $F_{j}$ of $\psi$, there may exist more than one common edge. Thus, between two vertices $x_{i}$ and $x_{j}$ in fuzzy dual graph $\psi^{\prime}$, there may be more than one edge. We denote $\left(x_{i}, x_{j}\right)_{\nu^{l}}$ be the membership value of the $l$-th edge between $x_{i}$ and $x_{j}$. The membership values of the fuzzy edges of the fuzzy dual graph are given by $\left(x_{i}, x_{j}\right)_{\nu^{l}}=(u, v)_{\mu^{j}}^{l}$ where $(u, v)^{l}$ is an edge in the boundary between two strong fuzzy faces $F_{i}$ and $F_{j}$ and $l=1,2, \ldots, s$, where $s$ is the number of common edges in the boundary between $F_{i}$ and $F_{j}$ or the number of edges between $x_{i}$ and $x_{j}$.

If there be any strong pendant edge in the 0.67 -fuzzy planar graph, then there will be a self loop in $\psi^{\prime}$ corresponding to this pendant edge. The edge membership value of the self loop is equal to the membership value of the pendant edge.

Fuzzy dual graph of 0.67 -fuzzy planar graph does not contain point of intersection of edges for a certain representation, so it is 0.67 -fuzzy planar graph with planarity value 1 . Thus the fuzzy face of fuzzy dual graph can be similarly described as in 0.67 -fuzzy planar graphs. 
Example 3: In Fig. 3, a 0.67-fuzzy planar graph $\psi=$ $(V, \sigma, E)$ where $V=\{a, b, c, d\}$ is given. For this graph let $\sigma(a)=0.6, \sigma(b)=0.7, \sigma(c)=0.8, \sigma(d)=0.9$.

and $E=\{((a, b), 0.5),((a, c), 0.4),((a, d), 0.55)$,

$((b, c), 0.45),((c, d), 0.7)\}$.

Thus, the 0.67-fuzzy planar graph has the following fuzzy faces

$F_{1}$ (bounded by $\left.((a, b), 0.5),((a, c), 0.4),((b, c), 0.45)\right)$,

$F_{2}$ (bounded by $\left.((a, d), 0.55),((c, d), 0.7),((a, c), 0.4)\right)$,

and outer fuzzy face

$F_{3}$ (surrounded by $\left.((a, b), 0.5),((b, c), 0.45),((c, d), 0.7),((a, d), 0.55)\right)$.

The fuzzy dual graph is constructed as follows. Here all the fuzzy faces are strong fuzzy faces. For each strong fuzzy face, we consider a vertex for the fuzzy dual graph. Thus the vertex set $V^{\prime}=\left\{x_{1}, x_{2}, x_{3}, x_{4}\right\}$ where the vertex $x_{i}$ is taken corresponding to the strong fuzzy face $F_{i}$, $i=1,2,3,4$. So $\sigma^{\prime}\left(x_{1}\right)=\max \{0.5,0.4,0.45\}=0.5$, $\sigma^{\prime}\left(x_{2}\right)=\max \{0.55,0.7,0.4\}=0.7, \sigma^{\prime}\left(x_{3}\right)$

$=\max \{0.5,0.45,0.7,0.55\}=0.7$.

There are two common edges $(a, d)$ and $(c, d)$ between the faces $F_{2}$ and $F_{3}$ in $\psi$. Hence between the vertices $x_{2}$ and $x_{3}$, two edges exist in the fuzzy dual graph of $\psi$. Here membership values of these edges are given by $\left(x_{2}, x_{4}\right)_{\nu^{1}}=(c, d)_{\mu^{1}}=0.7$, $\left(x_{2}, x_{4}\right)_{\nu^{2}}=(a, d)_{\mu^{1}}=0.55$.

The membership values of other edges of the fuzzy dual graph are calculated as $\left(x_{1}, x_{2}\right)_{\nu^{1}}=(a, c)_{\mu^{1}}=0.4$, $\left(x_{1}, x_{3}\right)_{\nu^{1}}=(a, b)_{\mu^{1}}=0.5,\left(x_{1}, x_{3}\right)_{\nu^{2}}=(b, c)_{\mu^{1}}=0.45$.

Thus the edge set of fuzzy dual graph is $E^{\prime}=$ $\left\{\left(\left(x_{1}, x_{2}\right), 0.4\right),\left(\left(x_{1}, x_{3}\right), 0.5\right),\left(\left(x_{1}, x_{3}\right), 0.45\right)\right.$,

$\left.\left(\left(x_{2}, x_{3}\right), 0.7\right),\left(\left(x_{2}, x_{3}\right), 0.55\right)\right\}$.

In Fig. 3, the fuzzy dual graph $\psi^{\prime}=\left(V^{\prime}, \sigma^{\prime}, E^{\prime}\right)$ of $\psi$ is drawn by dotted line.

Theorem 4: Let $\psi$ be a 0.67 -fuzzy planar graph without weak edges. The number of vertices, number of fuzzy edges and number of strong faces of $\psi$ are denoted by $n, p, m$ respectively. Also let $\psi^{\prime}$ be the fuzzy dual graph of $\psi$. Then

(i) the number of vertices of $\psi^{\prime}$ is equal to $m$,

(ii) number of edges of $\psi^{\prime}$ is equal to $p$,

(iii) number of fuzzy faces of $\psi^{\prime}$ is equal to $n$.

Proof. Proof of (i), (ii) and (iii) are obvious from the definition of fuzzy dual graph.

Theorem 5: Let $\psi^{\prime}$ be a fuzzy dual graph of a 0.67 -fuzzy planar graph $\psi$. The number of strong fuzzy faces in $\psi^{\prime}$ is less than or equal to the number of vertices of $\psi$.

Proof. Here $\psi^{\prime}$ is a fuzzy dual graph of a 0.67 -fuzzy planar graph $\psi$. Let $\psi$ has $n$ vertices and $\psi^{\prime}$ has $m$ strong fuzzy faces. Now, $\psi$ may have weak edges and strong edges. To construct fuzzy dual graph, weak edges are to eliminate. Thus if $\psi$ has some weak edges, some vertices may have all its adjacent edges as weak edges. Let the number of such vertices be $t$. These vertices are not bounding any strong fuzzy faces. If we remove these vertices and adjacent edges, then the number of vertices is $n-t$. Again, from Theorem $4, m=n-t$. Hence, in general $m \leq n$. This concludes that the number of strong fuzzy faces in $\psi^{\prime}$ is less than or equal to the number of vertices of $\psi$.

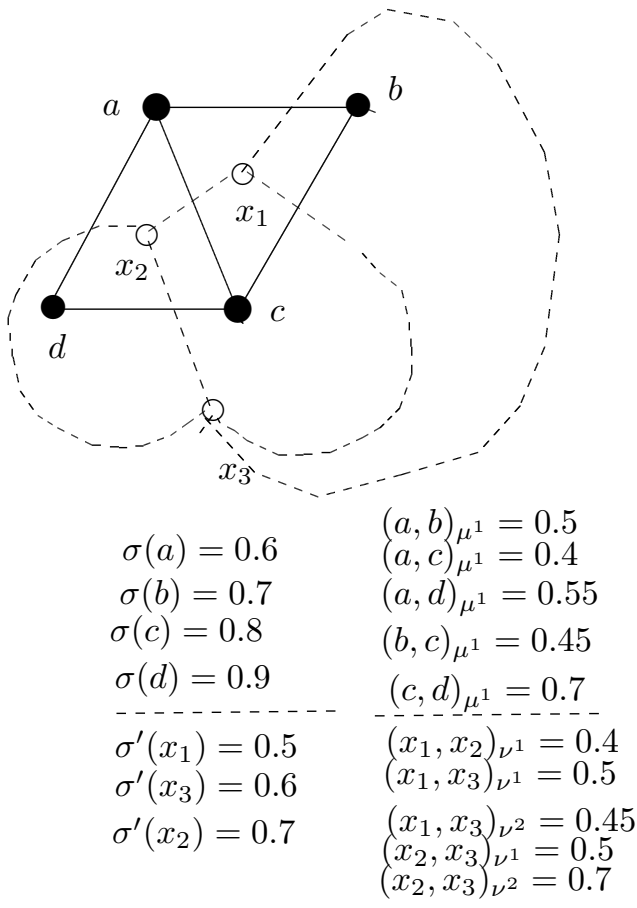

Fig. 3. Example of fuzzy dual graph.

An example is considered to illustrate the statement. Let $\psi=(V, \sigma, E)$ be a 0.67 -fuzzy planar graph where $V=$ $\{a, b, c, d\} . \sigma(a)=0.8, \sigma(b)=0.7, \sigma(c)=0.9, \sigma(d)=0.3$. $E=\{((a, b), 0.7),((b, c), 0.7),((c, d), 0.2),((b, d), 0.2)$,

$((a, d), 0.2)\}$. The corresponding fuzzy dual graph is $\psi^{\prime}=\left(V^{\prime}, \sigma^{\prime}, E^{\prime}\right)$ where $V^{\prime}=\left\{x_{1}, x_{2}, x_{3}\right\}$. $\sigma^{\prime}\left(x_{1}\right)=0.7, \sigma^{\prime}\left(x_{2}\right)=0.7, \sigma^{\prime}\left(x_{3}\right)=0.7$. $E^{\prime}=\left\{\left(\left(x_{1}, x_{2}\right), 0.2\right),\left(\left(x_{1}, x_{3}\right), 0.2\right),\left(\left(x_{1}, x_{3}\right), 0.7\right)\right.$,

$\left.\left(\left(x_{2}, x_{3}\right), 0.2\right),\left(\left(x_{2}, x_{3}\right), 0.7\right)\right\}$. Here number of strong fuzzy face is one while number of fuzzy face is three (see Fig. 4).

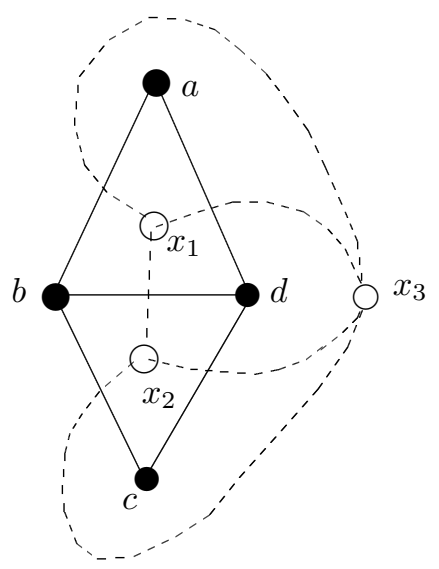

Fig. 4. Example of a fuzzy dual graph with strong face.

Theorem 6: Let $\psi=(V, \sigma, E)$ be a 0.67 -fuzzy planar graph without weak edges and the fuzzy dual graph of $\psi$ be $\psi^{\prime}=\left(V^{\prime}, \sigma^{\prime}, E^{\prime}\right)$. The membership values of fuzzy edges of 
$\psi^{\prime}$ are equal to membership values of the fuzzy edges of $\psi$. Proof. Let $\psi=(V, \sigma, E)$ be a 0.67 -fuzzy planar graph without weak edges. The fuzzy dual graph of $\psi$ is $\psi^{\prime}=\left(V^{\prime}, \sigma^{\prime}, E^{\prime}\right)$ which is a 0.67 -fuzzy planar graph as there is no point of intersection between any edges. Let $\left\{F_{1}, F_{2}, \ldots, F_{k}\right\}$ be the set of strong fuzzy faces of $\psi$.

From the definition of fuzzy dual graph we know that $\left(x_{i}, x_{j}\right)_{\nu^{l}}=(u, v)_{\mu^{j}}^{l}$ where $(u, v)^{l}$ is an edge in the boundary between two strong fuzzy faces $F_{i}$ and $F_{j}$ and $l=1,2, \ldots, s$, where $s$ is the number of common edges in the boundary between $F_{i}$ and $F_{j}$.

The numbers of fuzzy edges of two fuzzy graphs $\psi$ and $\psi^{\prime}$ are same as $\psi$ has no weak edges. For each fuzzy edge of $\psi$ there is a fuzzy edge in $\psi^{\prime}$ with same membership value.

\section{ISOMORPHISM ON FUZZY PLANAR GRAPHS}

Isomorphism between fuzzy graphs is an equivalence relation. But, if there is an isomorphism between two fuzzy graph and one is fuzzy planar graph, then the other will be fuzzy planar graph. This result is proved in the following theorem.

Theorem 7: Let $\psi$ be a fuzzy planar graph. If there exists an isomorphism $h: \psi \rightarrow \xi$ where $\xi$ is a fuzzy graph, $\xi$ can be drawn as fuzzy planar graph with same planarity value of $\psi$.

Proof. Let $\psi$ be a fuzzy planar graph and there exists an isomorphism $h: \psi \rightarrow \xi$ where $\xi$ is a fuzzy graph. Now, isomorphism preserves edge and vertex weights. Also the order and size of fuzzy graphs are preserved in isomorphic fuzzy graphs [20]. So, the order and size of $\xi$ will be equal to $\psi$. Then, $\xi$ can be drawn similarly as $\psi$. Hence, the number of intersection between edges and fuzzy planarity value of $\xi$ will be same as $\psi$. This concludes that $\xi$ can be drawn as fuzzy planar graph with same fuzzy planarity value.

In crisp graph theory, dual of dual graph of a planar graph is planar graph itself. In fuzzy graph concept, fuzzy dual graph of a fuzzy dual graph is not isomorphic to fuzzy planar graph. The membership values of vertices of fuzzy dual graph are the maximum membership values of its bounding edges of the corresponding fuzzy faces in fuzzy planar graph. Thus vertex weight is not preserved in fuzzy dual graph. But edge weight is preserved in fuzzy dual graph. This result is established in following theorem.

Theorem 8: Let $\psi_{2}$ be the fuzzy dual graph of fuzzy dual graph of a 0.67 -fuzzy planar graph $\psi$ without weak edges. Then there exists a co-weak isomorphism between $\psi$ and $\psi_{2}$. Proof. Let $\psi$ be a 0.67 -fuzzy planar graph which has no weak edges. Also let, $\psi_{1}$ be the fuzzy dual graph of $\psi$ and $\psi_{2}$ be the fuzzy dual graph of $\psi_{1}$. Now we have to establish a co-weak isomorphism between $\psi_{2}$ and $\psi$. As the number of vertices of $\psi_{2}$ is equal to that of strong fuzzy faces of $\psi_{1}$. Again the number of strong fuzzy faces is equal to the number of vertices of $\psi$. Thus, the number of vertices of $\psi_{2}$ and $\psi$ are same. Also, the numbers of edges of a fuzzy planar graph and its dual graph are same. By the definition of fuzzy dual graph, the edge membership value of an edge in fuzzy dual graph is equal to the edge membership value of an edge in fuzzy planar graph. Thus we can construct a co-weak isomorphism from $\psi_{2}$ to $\psi$. Hence the result is true.

The Theorem 8 can be explained by the following example. Here a 0.67 -fuzzy planar graph $\psi$ is constructed (See Fig. 5(a)). Then its fuzzy dual graph $\psi_{1}$ is drawn in Fig. 5(b). Also the fuzzy dual graph $\psi_{2}$ of $\psi_{1}$ is drawn in Fig. 5(c). Now, we construct a bijective mapping from vertices of $\psi_{2}$ to vertices of $\psi$ as $a_{1} \rightarrow a, b_{1} \rightarrow b, c_{1} \rightarrow c, d_{1} \rightarrow d$. Similarly, we can extend the mapping from edge set of $\psi_{2}$ to the edge set of $\psi$. It is observed that the vertex membership values of $\psi_{2}$ is less than or equal to the vertex membership values of $\psi$ under the mapping and edge membership values are equal under the mapping. Thus the mapping is said to satisfy the co-weak isomorphism property.

Two fuzzy planar graphs with same number of vertices may be isomorphic. But, the relations between fuzzy planarity values of two fuzzy planar graphs may have the following relations.

Theorem 9: Let $\xi_{1}$ and $\xi_{2}$ be two isomorphic fuzzy graphs with fuzzy planarity values $f_{1}$ and $f_{2}$ respectively. Then $f_{1}=$ $f_{2}$.

The proof of the theorem is the immediate consequence of Theorem 7.

Theorem 10: Let $\xi_{1}$ and $\xi_{2}$ be two weak isomorphic fuzzy graphs with fuzzy planarity values $f_{1}$ and $f_{2}$ respectively. $f_{1}=$ $f_{2}$ if the edge membership values of corresponding intersecting edges are same.

Proof. Here $\xi_{1}=\left(V, \sigma_{1}, \mu_{1}\right)$ and $\xi_{2}=\left(V, \sigma_{2}, \mu_{2}\right)$ are two weak isomorphic fuzzy graphs with fuzzy planarity values $f_{1}$ and $f_{2}$ respectively. As two fuzzy graphs are weak isomorphic, $\sigma_{1}(x)=\sigma_{2}(y)$ for some $x$ in $\xi_{1}$ and $y$ in $\xi_{2}$. Let the graphs have one point of intersection. Let two intersecting edges be $\left(a_{1}, b_{1}\right)$ and $\left(c_{1}, d_{1}\right)$ in $\xi_{1}$. Also two corresponding edges in $\xi_{2}$ be $\left(a_{2}, b_{2}\right)$ and $\left(c_{2}, d_{2}\right)$. Then, intersecting value of the point is given by $\frac{\frac{\mu\left(a_{1}, b_{1}\right)}{\sigma\left(a_{1}\right) \wedge \sigma\left(b_{1}\right)}+\frac{\mu\left(c_{1}, d_{1}\right)}{\sigma\left(c_{1}\right) \sigma\left(d_{1}\right)}}{2}$. The intersecting value of the corresponding point in $\xi_{2}$ is given as $\frac{\frac{\mu\left(a_{2}, b_{2}\right)}{\sigma\left(a_{2}\right) \wedge \sigma\left(b_{2}\right)}+\frac{\mu\left(c_{2}, d_{2}\right)}{\sigma\left(c_{2}\right) \wedge \sigma\left(d_{2}\right)}}{2}$. Now, $f_{1}=f_{2}$, if $\mu\left(a_{1}, b_{1}\right)=\mu\left(a_{2}, b_{2}\right)$. The number of point of intersections may increase. But, if the sum of the intersecting value of $\xi_{1}$ is equal to that of $\xi_{2}$, fuzzy planarity values of the graphs must be equal. Thus, for equality of $f_{1}$ and $f_{2}$, the edge membership values of intersecting edges of $\xi$ are equal to the edge membership values of the corresponding edges in $\xi_{2}$.

Theorem 11: Let $\xi_{1}$ and $\xi_{2}$ be two co-weak isomorphic fuzzy graphs with fuzzy planarity values $f_{1}$ and $f_{2}$ respectively. $f_{1}=f_{2}$ if the minimum of membership values of the end vertices of corresponding intersecting edges are same.

Proof. Here $\xi_{1}=\left(V, \sigma_{1}, \mu_{1}\right)$ and $\xi_{2}=\left(V, \sigma_{2}, \mu_{2}\right)$ are two co-weak isomorphic fuzzy graphs with fuzzy planarity values $f_{1}$ and $f_{2}$ respectively. As two fuzzy graphs are co-weak isomorphic, $\mu_{1}(x, y)=\mu_{2}(z, t)$ for some edge $(x, y)$ in $\xi_{1}$ and $(z, t)$ in $\xi_{2}$. Let the graphs have one point of intersection. Let two intersecting edges be $\left(a_{1}, b_{1}\right)$ and $\left(c_{1}, d_{1}\right)$ in $\xi_{1}$. Also, two corresponding edges in $\xi_{2}$ be $\left(a_{2}, b_{2}\right)$ and $\left(c_{2}, d_{2}\right)$. Then, inter- 


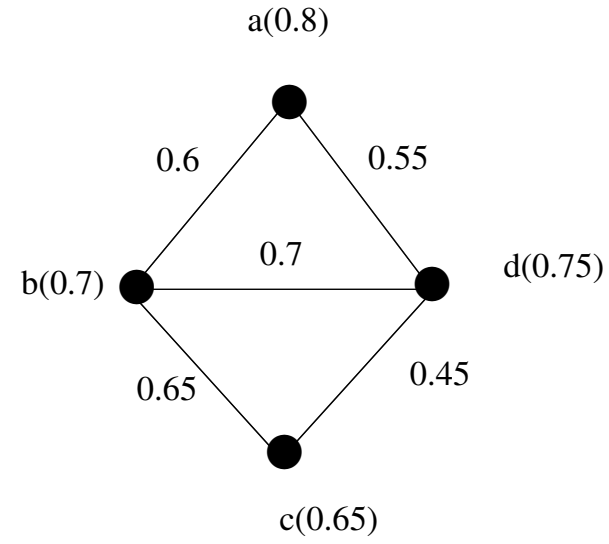

(a): A 0.67-fuzzy planar graph $\psi$

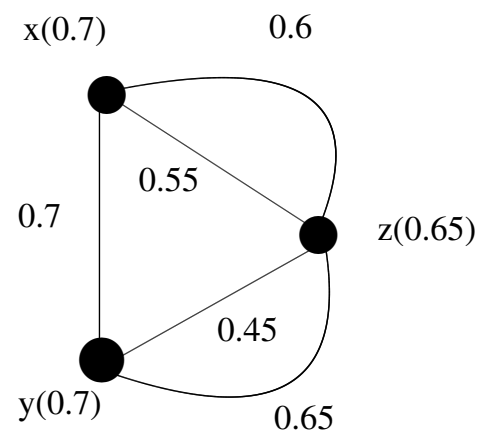

(b): Fuzzy dual graph $\psi_{1}$ of $\psi$

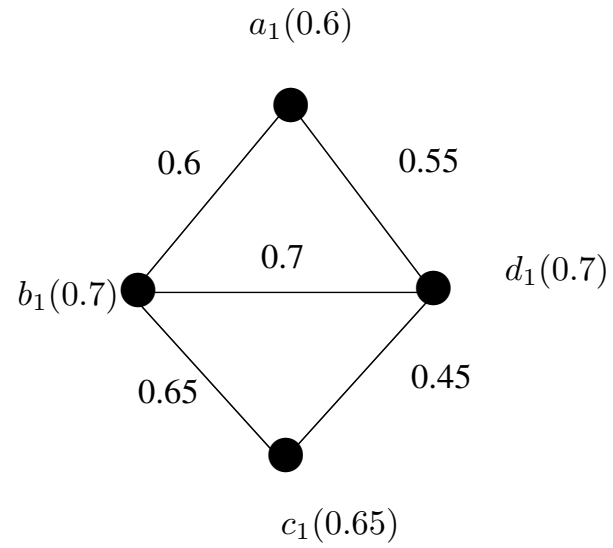

(c): Fuzzy dual graph $\psi_{2}$ of $\psi_{1}$

Fig. 5. Dual of dual is co-weak isomorphic to planar graph in fuzzy graph theory. secting value of the point is given by $\frac{\frac{\mu\left(a_{1}, b_{1}\right)}{\sigma\left(a_{1}\right) \wedge \sigma\left(b_{1}\right)}+\frac{\mu\left(c_{1}, d_{1}\right)}{\sigma\left(c_{1}\right) \wedge \sigma\left(d_{1}\right)}}{2}$. The intersecting value of the corresponding point in $\xi_{2}$ is given as $\frac{\frac{\mu\left(a_{2}, b_{2}\right)}{\sigma\left(a_{2}\right) \wedge \sigma\left(b_{2}\right)}+\frac{\mu\left(c_{2}, d_{2}\right)}{\sigma\left(c_{2}\right) \wedge \sigma\left(d_{2}\right)}}{2}$. Now, the fuzzy planarity values $f_{1}$ $=f_{2}$, if $\sigma_{1}\left(a_{1}\right) \wedge \sigma\left(b_{1}\right)=\sigma_{2}\left(a_{2}\right) \wedge \sigma_{2}\left(b_{2}\right)$. The number of point of intersections may increase. But if the sum of the intersecting value of $\xi_{1}$ is equal to that of $\xi_{2}$, fuzzy planarity values of the graphs must be equal. Thus, for equality of $f_{1}$ and $f_{2}$, the minimum membership value of end vertices of an edge in $\xi_{1}$ is equal to that of a corresponding edge in $\xi_{2}$.

\section{CONCLUSION}

This study describes the fuzzy multigraphs, fuzzy planar graphs, and a very important consequence of fuzzy planar graph known as fuzzy dual graphs. In crisp planar graph, no edge intersects other. In fuzzy graph, an edge may be weak or strong. Using the concept of weak edge, we define fuzzy planar graph in such a way that an edge can intersect other edge. But, this facility violates the definition of planarity of graph. Since the role of weak edge is insignificant, the intersection between a weak edge and an other edge is less important. Motivating from this idea, we allow the intersection of edges in fuzzy planar graph. It is well known that if the membership values of all edges become one, the graph becomes crisp graph. Keeping this idea in mind, we define a new term called fuzzy planarity value of a fuzzy graph. If the fuzzy planarity value of a fuzzy graph is one, then no edge crosses other edge. This leads to the crisp planar graph. Thus, the fuzzy planarity value measures the amount of planarity of a fuzzy graph. This is a very interesting concept of fuzzy graph theory. Strong fuzzy planar graphs and a distinguishable subclass of strong fuzzy planar graph namely 0.67-fuzzy planar graphs have been exemplified. From the definitions, it is concluded that 0.67 -fuzzy planar graph $\subset$ strong fuzzy planar graph $\subset$ fuzzy planar graph. Another important term of planar graph is "face" which is redefined in fuzzy planar graph. A particular type of fuzzy face called strong fuzzy face is incorporated. Besides, isomorphism properties of fuzzy planar graphs are investigated. It is shown that dual of dual fuzzy graphs are coweak isomorphism to fuzzy planar graph. Several properties of isomorphism on fuzzy planar graphs are explained. It may be noted that, in this article, fuzzy dual graph is defined for the 0.67-fuzzy planar graph. But, if the planarity value is less than 0.67 , then some modification is required to define dual graph. This is to be investigated in near future.

\section{REFERENCES}

[1] N. abdul-jabbar, J. H. Naoom and, E. H. Ouda, Fuzzy dual graph, Journal Of Al-Nahrain University, 12(4), 168-171, 2009.

[2] M. Akram, Bipolar fuzzy graphs, Information Science, 181, 5548-5564, 2011.

[3] M. Akram, Interval-valued fuzzy line graphs, Neural Computing \& Applications, 21, 145-150, 2012.

[4] M. Akram and W. A. Dudek, Intuitionistic fuzzy hypergraphs with applications, Information Sciences, 218, 182-193, 2013.

[5] M. Akram, Bipolar fuzzy graphs with applications, Knowledge Based Systems, 39, 1-8, 2013.

[6] V. K. Balakrishnan, Graph Theory, McGraw-Hill, 1997.

[7] K. R. Bhutani and A. Battou, On M-strong fuzzy graphs, Information Sciences, 155(12), 103-109, 2003. 
[8] K. R. Bhutani and A. Rosenfeld, Strong arcs in fuzzy graphs, Information Sciences, 152, 319-322, 2003.

[9] C. Eslahchi and B. N. Onaghe, Vertex strength of fuzzy graphs, International Journal of Mathematics and Mathematical Sciences, Volume 2006, Article ID 43614, Pages 1-9, DOI 10.1155/IJMMS/2006/43614.

[10] M. Fazzolari and H. Ishibuchi, A review of the application of multiobjective evolutionary fuzzy systems: current status and further directions, IEEE Transection on Fuzzy Systems, 21(1), 45-65, 2013.

[11] P. Ghosh, K. Kundu and D. Sarkar, Fuzzy graph representation of a fuzzy concept lattice, Fuzzy Sets and Systems, 161(12), 1669-1675, 2010.

[12] L. T. Koczy, Fuzzy graphs in the evaluation and optimization of networks, Fuzzy Sets and Systems, 46, 307-319, 1992.

[13] Q. Liang and J. M. Mendel, MPEG VBR video traffic modelling and classification using fuzzy technique, IEEE Transection on Fuzzy Systems, 9(1), 183-193, 2001.

[14] K. -C. Lin and M. -S. Chern, The fuzzy shortest path problem and its most vital arcs, Fuzzy Sets and Systems, 58, 343-353, 1993.

[15] S. Mathew and M.S. Sunitha, Types of arcs in a fuzzy graph, Information Sciences, 179, 1760-1768, 2009.

[16] S. Mathew and M.S. Sunitha, Strongest strong cycles and theta fuzzy graphs, IEEE Transections on Fuzzy Systems, 2013.

[17] M. L. N. McAllister, Fuzzy intersection graphs, Comput. Math. Applic., 15 (10), 871-886, 1988.

[18] J. N. Mordeson and P. S. Nair, Fuzzy Graphs and Hypergraphs, Physica Verlag, 2000.

[19] S. Munoz, M. T. Ortuno, J. Ramirez and J. Yanez, Coloring fuzzy graphs, Omega, 33(3), 211-221, 2005.

[20] A. Nagoorgani and J. Malarvizhi, Isomorphism on fuzzy graphs, World Academy of Science, Engineering and Technology, 23, 505-511, 2008.

[21] A. Nagoorgani and R. J. Hussain, Fuzzy effective distance $K$ dominating sets and their applications, International Journal of Algorithms, Computing and Mathematics, 2(3), 25-36, 2009.

[22] G. Nirmala and K. Dhanabal, Special planar fuzzy graph configurations, International Journal of Scientific and Research Publications, 2(7), 1-4, 2012.

[23] A. Rosenfeld, Fuzzy graphs, in: L.A. Zadeh, K.S. Fu, M. Shimura (Eds.), Fuzzy Sets and Their Applications, Academic Press, New York, 77-95, 1975.

[24] S. Samanta and M. Pal, Fuzzy $k$-competition graphs and $p$-competition fuzzy graphs, Fuzzy Engineering and Information, 5(2), 191-204, 2013.

[25] S. Samanta and M. Pal, Fuzzy tolerance graphs, International Journal of Latest Trends in Mathematics, 1(2), 57-67, 2011.

[26] S. Samanta and M. Pal, Fuzzy threshold graphs, CIIT International Journal of Fuzzy Systems, 3(12), 360-364, 2011.

[27] S. Samanta and M. Pal, Irregular bipolar fuzzy graphs, Inernational Journal of Applications of Fuzzy Sets, 2, 91-102, 2012.

[28] S. samanta and M. Pal, Bipolar fuzzy hypergraphs, International Journal of Fuzzy Logic Systems, 2(1), 17 - 28, 2012.

[29] S. Samanta, M. Pal and A. Pal, Some more results on bipolar fuzzy sets and bipolar fuzzy intersection graphs, To appear in The Journal of Fuzzy Mathematics.

[30] S. Samanta and M. Pal, A new approach to social networks based on fuzzy graphs, To appear in Journal of Mass Communication and Journalism.

[31] R. R. Yager, On the theory of bags, Int. J. General Systems, 13, 23-37, 1986. 